\title{
Imaging biomarkers for the diagnosis of Prion disease
}

\section{Liane S. Canas, Benjamin Yvernault, Carole Sudre, Enrico De Vita, M. Jorge Cardoso, et al.}

Liane S. Canas, Benjamin Yvernault, Carole Sudre, Enrico De Vita, M. Jorge Cardoso, John Thornton, Frederik Barkhof, Sébastien Ourselin, Simon Mead, Marc Modat, "Imaging biomarkers for the diagnosis of Prion disease," Proc. SPIE 10574, Medical Imaging 2018: Image Processing, 1057405 (2 March 2018); doi: 10.1117/12.2293676

SPIE. Event: SPIE Medical Imaging, 2018, Houston, Texas, United States 


\title{
Imaging biomarkers for the diagnosis of Prion disease
}

\author{
Liane S Canas ${ }^{\mathrm{a}^{*}}$, Benjamin Yvernault ${ }^{\mathrm{a}}$, Carole Sudre ${ }^{\mathrm{a}, \mathrm{b}}$, Enrico De Vita ${ }^{\mathrm{c}}$, M Jorge Cardoso ${ }^{\mathrm{a}, \mathrm{b}}$, \\ John Thornton ${ }^{\mathrm{c}}$, Frederik Barkhof ${ }^{\mathrm{a}, \mathrm{d}}$, Sébastien Ourselina, b, Simon Mead ${ }^{\mathrm{e}}$, and Marc \\ Modat ${ }^{\mathrm{a}, \mathrm{b}}$ \\ ${ }^{a}$ Translational Imaging Group, Centre for Medical Image Computing, University College \\ London, London, UK \\ ${ }^{\mathrm{b}}$ Dementia Research Centre, UCL Institute of Neurology, London, UK \\ 'Lysholm Department of Neuroradiology, National Hospital for Neurology and Neurosurgery, \\ London, UK \\ ${ }^{\mathrm{d} I n s t i t u t e}$ of Neurology, University College London, UK \\ ${ }^{\mathrm{e}} \mathrm{MRC}$ Prion Unit, Department of Neurodegenerative Disease, UCL Institute of Neurology, \\ London, UK
}

\begin{abstract}
Prion diseases are a group of progressive neurodegenerative conditions which cause cognitive impairment and neurological deficits. To date, there is no accurate measure that can be used to diagnose this illness, or to quantify the evolution of symptoms over time. Prion disease, due to its rarity, is in fact commonly mistaken for other types of dementia. A robust tool to diagnose and quantify the progression of the disease is key as it would lead to more appropriately timed clinical trials, and thereby improve patients' quality of life.

The approaches used to study other types of neurodegenerative diseases are not satisfactory to capture the progression of human form of Prion disease. This is due to the large heterogeneity of phenotypes of Prion disease and to the lack of consistent geometrical pattern of disease progression.

In this paper, we aim to identify and select imaging biomarkers that are relevant for the diagnostic on Prion disease. We extract features from magnetic resonance imaging data and use genetic and demographic information from a cohort affected by genetic forms of the disease. The proposed framework consists of a multi-modal subjectspecific feature extraction step, followed by a Gaussian Process classifier used to calculate the probability of a subject to be diagnosed with Prion disease. We show that the proposed method improves the characterisation of Prion disease.
\end{abstract}

Keywords: Prion diseases, Creutzfeldt-Jakob disease, Imaging biomarkers, Diagnosis, Prognosis

\section{DESCRIPTION OF PURPOSE}

Prion diseases are a group of progressive neurodegenerative conditions which cause cognitive impairment and neurological deficits. The human form of prion disease can be separated in three forms: sporadic, acquired and inherited. The most common human form is the Creutzfeldt-Jakob disease (CJD). ${ }^{1}$ All cases of CJD are characterised by a high rate of progression: the expected time of survival after diagnosis is typically less that 12 months from diagnosis. ${ }^{2}$ The inherited human form of Prion disease (IPD) occurs as a result of one of more than 30 mutations in the prion protein gene $(P R N P)$, hence conferring various phenotypes to the disease, yielding to high heterogeneity. Variablity has also been reported among affected individuals within families carrying the same mutation..$^{3,4}$

Due to the heterogeneity of observed phenotypes, the clinical diagnosis of CJD is a challenging task while the patient is alive, particularly in the earlier stages of the disease. ${ }^{1}$ In practice, some cases of CJD are routinely mistaken for other common forms of dementia, which results in CJD being under diagnosed. ${ }^{5}$ In the clinic, the

\footnotetext{
* Further author information: liane.canas.15@ucl.ac.uk
} 


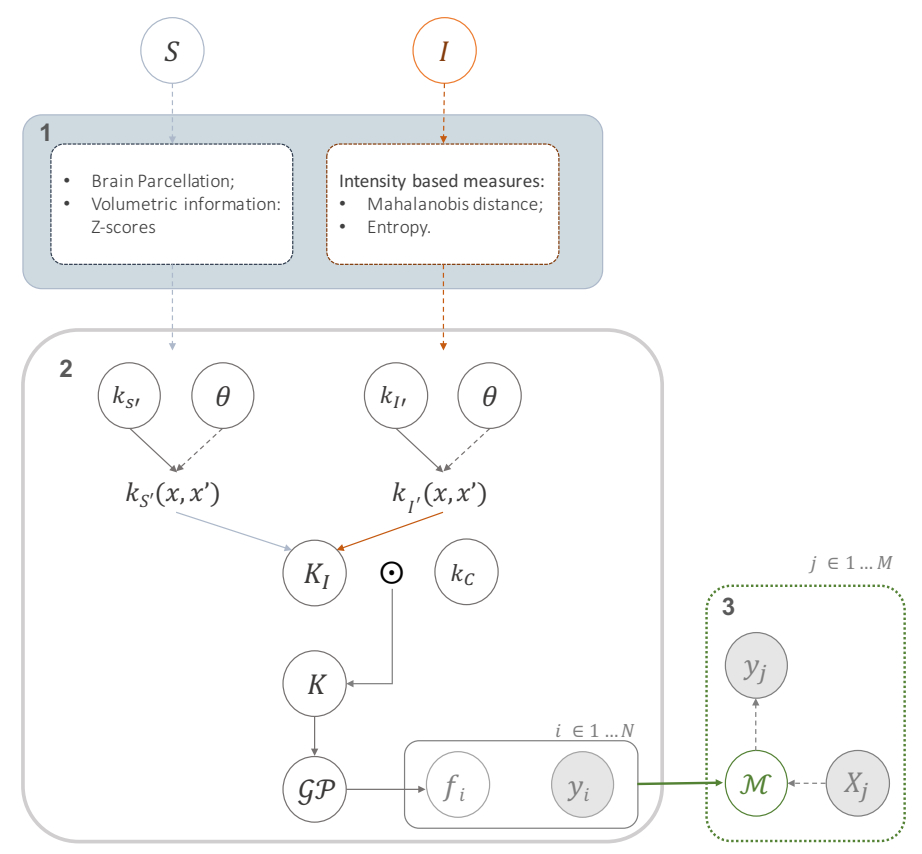

Figure 1. Graphical representation of the framework. $\mathcal{K}$ is result of the addition of kernels correspondent to each MRI modality. $f$ denotes the latent function, whereas $y$ is the values predicted by using the estimated latent function. The subscripts $i$ denote sampled latent values for each point to a maximum of $N$ data points. $\mathcal{M}$ is the model selected and used to estimate $y_{j}$ predictions of $j$ observation given a set of features $\mathbf{X}_{j}$.

diagnostic of CJD relies on the visual read of Magnetic Resonance Imaging (MRI) scans, as they present signal abnormalities such as hyperintensities in FLAIR and DWI images. ${ }^{6,7}$

To this date, a definite diagnosis of CJD is only possible post mortem (brain autopsy). ${ }^{1}$ The lack of quantitative biomarkers, as well as the difficulty to accurately identify the onset of the disease and the fast rate of progression of CJD, have limited the clinical understanding of the progression of $\mathrm{CJD}^{5,8}$ and the development of automated tool for diagnostic and prognosis.

In an attempt to overcome the aforementioned issues, research is directed towards identifying the right biomarkers that characterise and discriminate the illness. If successful, an accurate knowledge of the disease progression steps and the anticipation of symptoms will allow more suitable clinical trials as well as better care of the patients' symptoms.

The proposed framework aims to (i) extract quantitative imaging biomarkers from MR images and (ii) show that those are fit for the diagnosis of CJD in its early stages. We also evaluate the advantages of using a Gaussian Process classifier (GP) to explain the interactions between several imaging biomarkers extracted from different MRI pulse-sequences.

\section{METHODS}

Our approach, graphically represented in figure 1, is composed of three main sections: (1) feature extraction followed by feature selection, (2) model training - estimation of the hyperparameters associated to the covariance kernel functions defined for the modalities under consideration -, and (3) subjects diagnostic.

\subsection{Features Extraction and Selection}

We considered two types of features, referred to in this paper as volumetric and intensity-based features. 


\subsubsection{Features Extraction}

Volumetric features were extracted from T1-weighted MRI scans (T1w) using the Geodesical Information Flows ${ }^{9}$ (GIF) algorithm. In brief, using multi-atlas segmentation propagation, GIF parcellates the brain into multiple regions of interest. From the obtained parcellation, a volumetric information is obtained for each individual brain region. To compensate for inter-subject normal variability, we regress the impact of volumetric confounding effects, such as age and total intracranial volume (TIV).

Intensity-based features were extracted from FLAIR images in order to rely on the same information that is used when doing a visual read in clinical practice. Indeed, we here aimed at automatically identifying regions of the brain that are affected by signal abnormality induced by CJD. ${ }^{10}$ We use a Gaussian Mixture Model (GMM) approach to model the intensities of the FLAIR scans. Using the Bayesian Model Selection algorithm (BaMoS), ${ }^{11}$ we segmented the normal and abnormal appearing tissue types (grey matter and white matter). Assuming that CJD does not cause lesions in the white matter tissue, we computed the Mahalanobis distance between the normal appearing white matter intensity distribution and the grey matter intensities for each region of interest as defined by GIF. We use the Mahalanobis distance enabled to compensate for possible variation in acquisition protocol since only the normal appearing white matter was used to normalise the distance. The median Mahalanobis distance per brain region of interest was used as a features with the assumption that the larger the amount of hyper-intensity in a given region of interest, the larger the distance.

The resulting features extracted from MR images are illustrated in Figure 2.
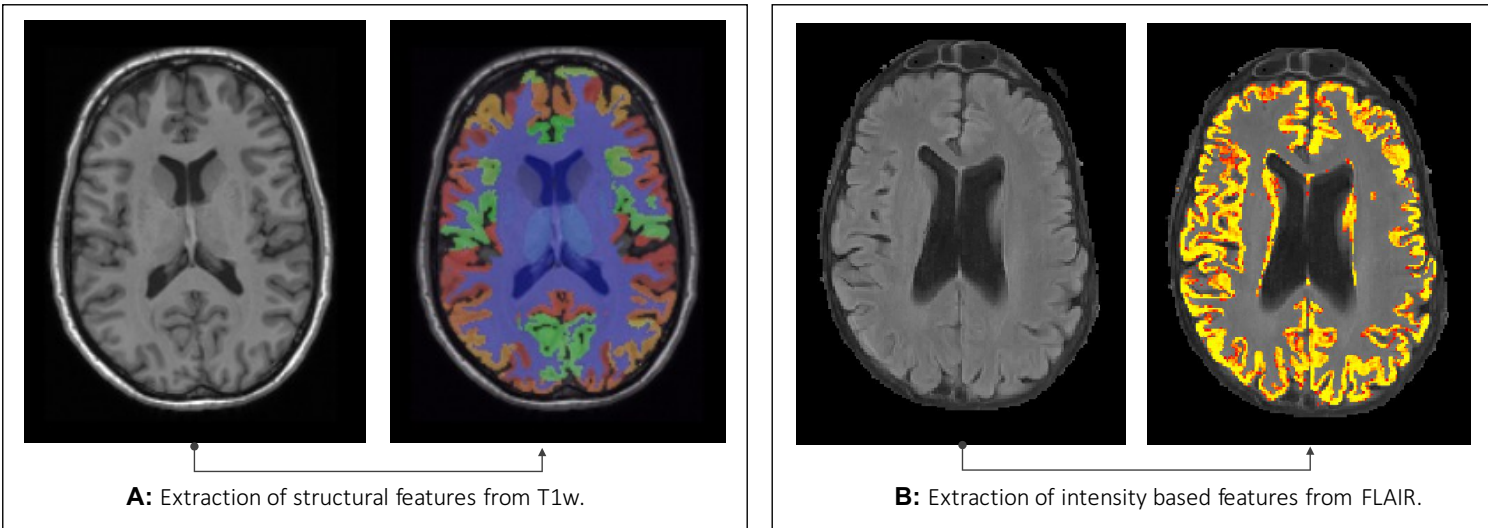

Figure 2. Imaging biomarkers extracted from the MR images. Left: Brain regions extracted using GIF [5]. Right: Mahalanobis distance between GM and WM computed per voxel. Higher values of Mahalanobis distance are represented by the red colour, whereas lower values are yellow.

\subsubsection{Features Selection}

In the literature, common approaches for classification usually rely on a consistent set of features across subjects. This can be done since most neurodegenerative diseases follow a common geometrical pattern across subjects and across time. However, these methods cannot be applied to CJD due to the high heterogeneity of the disease, where there is no consistent spatial pattern of disease progression.

We here hypothese that the disease does not follow a geometrical pattern but instead that imaging biomarkers can become abnormal in any location in the brain. The quantity of abnormality rather than its location is thus use to quantify the progression of the disease. To characterise the amount of abnormality of signal (volume or intensity), we convert the aforementioned volumetric and intensity features into z-scores by comparison with measurements obtained from a population of healthy subjects. The z-score values are then ranked per pulsesequence (structural, FLAIR) and only the highest values for each sequence are considered for subsequent learning and inference stages. As a consequence, only regions of the brain that most differ from the healthy control sample are kept for each subject, and the resulting sets of features are patient-specific. 


\subsection{Model learning}

Assuming that the inter-modality relationship can be modelled as a multi-task paradigm - a contribution of independent functions that explain the biomarkers progression - we implemented an Additive Gaussian process to perform the diagnosis of the subjects.

We implemented a non-parametric kernel-based model as follow:

$$
\mathcal{M}: y=f(X)+\varepsilon, f \sim \mathcal{G P}\left(\mu_{f} ; K+I \sigma_{f}\right), \varepsilon \sim \mathcal{N}\left(\mu_{\varepsilon} ; \sigma\right)
$$

This model was used to infer the subjects status, $y$, given a set of biomarkers $\mathbf{X} \in \mathcal{X}$ features space, composed of the volumetric and intensity-based features extracted from T1w and FLAIR images respectively.

The function $f$ describes the variance of the features, which explains the response variable $y$. By implementing a GP with $\mu_{f}=0$ and covariance kernel function $K$, we determine the pattern of the inductive generalization of the features under consideration. We use an addition of two linear logistic kernel functions to describe the evolution of the biomarkers between the two states of the disease: 0 - healthy control, 1 - symptomatic subjects. Further, the estimation of $\hat{y}_{i, b}$ requires to find the best hyperparameters of each kernel. The hyperparameters $\theta$ of the kernel functions are estimated via the maximisation of the marginal likelihood of the model, $p(y \mid X, \theta)$, as described in equation 1; i.e., the marginalisation over the kernel parameters is performed by maximum $a$ posteriori algorithm (MAP), and that the hyperparameters $\theta$ are estimated by bootstrapping.

$$
\{\hat{\theta}, \hat{\sigma}\}=\operatorname{argmax}_{\sigma, \theta} p(\theta, \sigma \mid \mathcal{M})=\operatorname{argmin}_{\sigma, \theta}[-\log p(\mathcal{M} \mid \theta, \sigma)+\log p(\theta, \sigma)]
$$

Note that our model also accounts for the individualised pattern of each genetic mutation of the genetic form of CJD. In order to reduce the bias introduced by the high number of genetic mutations, we group the subjects in three clusters according to the rate of disease progression associated with each mutation. This information is included in our model using a third kernel matrix, $K_{c}$. The matrix $K_{I}$, which encodes the imaging biomarker, is obtained by the addition of the two kernel matrices computed individually using the information extracted from the two MR modalities. The final matrix $K$ considered in the GP model results from the Hadamard product, $K_{c} \odot K_{I}$.

Finally, the classification of a new subject is given by the features extracted from section 2.1 and the model estimated in section 2.2 of this paper. Using Bayes theorem we predicted the probability of a subject to be either symptomatic or asymptomatic given the class-conditional distributions $p(X \mid y)$ fory $=0,1$, where 0 denotes the asymptomatic or healthy controls and 1 denotes the symptomatic subjects.

\section{RESULTS}

We extracted volumetric and intensity-based biomarkers from a dataset that embeds multiple patient subgroups: healthy controls, asymptomatic, converter and symptomatic subjects. The asymptomatic are defined as subjects who are carrying a genetic variant of the disease but do not manifest any clinical symptoms yet. The converter subjects are those that start exhibiting clinical symptom within a year after the MRI acquisition. The symptomatic subjects are those who have been scanned after the clinical onset of the disease. They consist of subjects with the sporadic and the inherited form of the disease.

Figure 3, top, shows that the volumetric features provide a good differentiation between the subgroups and that the level of abnormality increases as the disease progress over time.

To evaluate the reliability of the features extracted to characterise C.JD, we classified the symptomatic subjects using clinical and imaging data of a Prion diseases dataset that includes the baseline scans for both symptomatic and asymptomatic subjects. For the classification purposes, we exclude the sporadic CJD patients from our sample, due to the fact that these patients do not carry a genetic mutation, and the clinical manifestations of CJD progress faster when compared with inherited Prion disease patients. Therefore, our sample consists of 25 controls, 18 symptomatic subjects and 33 asymptomatic.

We divided our sample in to two sub-samples: a training set that corresponds to $70 \%$ of the symptomatic, asymptomatic and controls and the testing set that comprises of the remaining $30 \%$. In the current experimental 

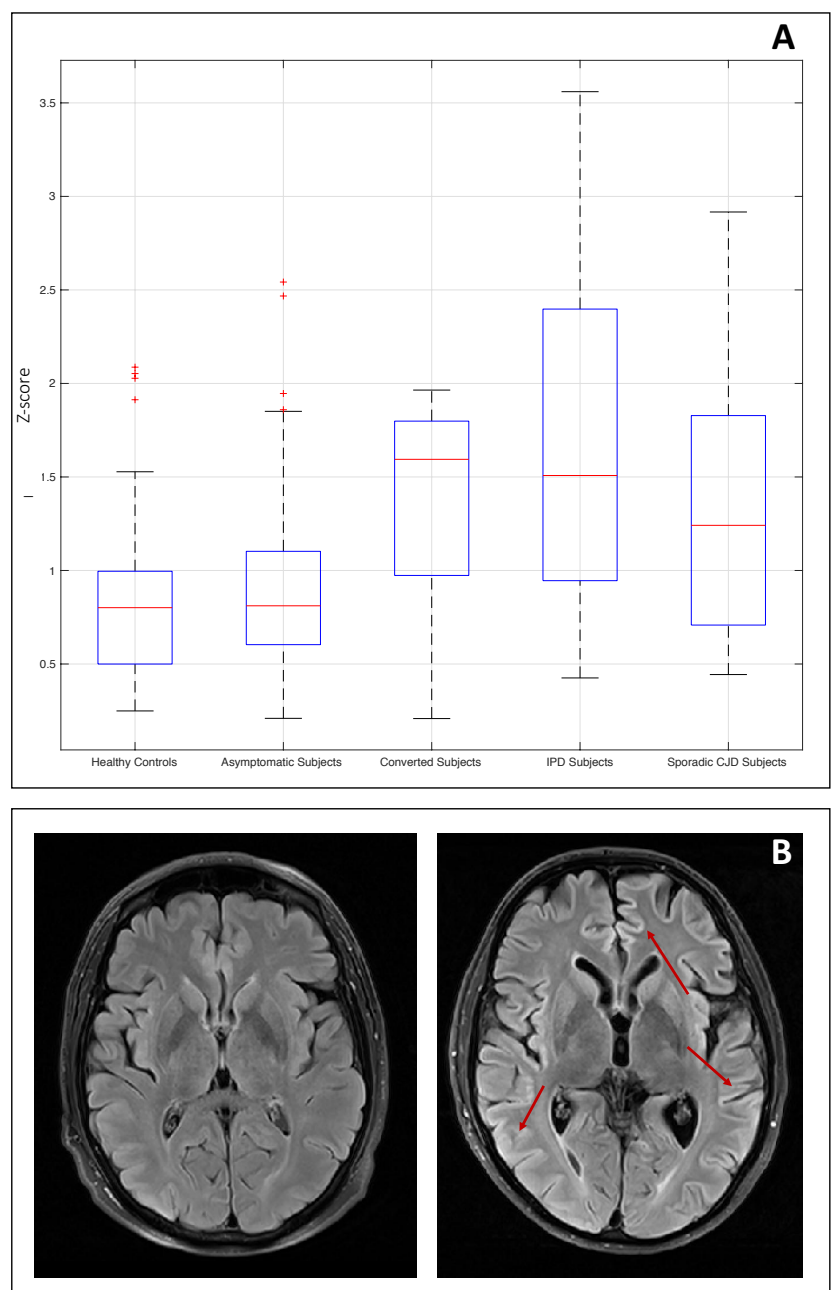

Figure 3. Imaging biomarkers used as features in the model. A - Volumetric features extracted from the T1w images. The 10 most abnormal brain region per subject are selected as features. B - Example of the intensity based features used in the model. The healthy control scan (left) shows a reduced number of hyperintensities the cortical grey matter when compared with a inherited CJD patient (right). The red arrows identify some of the lesions in the cortical grey matter.

set up we define as controls the asymptomatic subjects. Due to the relatively small sample size of our dataset, we employed a 10-fold cross-validation, and the mean accuracy was used to evaluate the model. We also ran our analysis with a logistic regression (LR) approach to assess the added value of using a GP.

Results show, in table 1, that the biomarkers extracted from T1w and FLAIR images have the potential to be used as features in a model developed to classify subjects with CJD. Using respectively GP and LR for classification, the subjects were correctly classified with $86 \%$ and $79 \%$ of accuracy. The results suggest that the proposed GP is more accurate for diagnosis; although, it has shown lower sensitivity in the identification of symptomatic subjects among healthy controls.

Table 1. Evaluation of the two methods used to diagnose CJD using the framework design to extract and select meaningful biomarkers from MRI modalities. We compare the results obtained using Logistic Regression (LR) and Gaussian Process (GP).

\begin{tabular}{llll}
\hline Approach & Balanced Accuracy & Recall & F1 Score \\
\hline LR & 0.787 & 0.914 & 0.903 \\
GP & 0.860 & 0.812 & 0.798
\end{tabular}




\section{DISCUSSION}

We developed a framework to extract imaging biomarkers especially relevant for the detection of CJD. We demonstrated that it is possible to use these biomarkers as inputs to a classification tool such as a Gaussian Process or a logistic regression. Indeed, the extracted biomarkers have shown promising results for the identification of inherited Prion disease symptomatic subjects against healthy controls and asymptomatic patients.

We hope that in the future, such approach will be beneficial to improve the rate of correct diagnostic of patient with Prion disease. The initial symptoms of the disease are usually mistaken for depression symptoms, and even in later stages of the disease, the symptoms are often considered as dementia symptoms, particularly in the inherited form of CJD, for which the progression is slower than the sporadic form.

In the future, we will apply the framework to predict when an asymptomatic subject is expected to start showing clinical symptoms. To do so, we intent to alter our model in several aspects. First, we are going to include sporadic cases to increase the available sample size. Second, we will include features from other sources. For example, diffusion weighted images is often used via qualitative read for diagnostic purposes. We should thus be able to extract a relevant imaging biomakers. Lastly, we are going to include longitudinal information that should enable use to better quantify how the abnormality of a given region if evolving over time.

Our second aim will be to investigate how the features we presented in this paper can be use for differential diagnostic rather than for a simpler disease versus control case. We thus would like to assess how we will be able to identify subjects with Prion disease from subjects with more common neurogenerative diseases such as Alzheimer's disease, fronto-temporal dementia or vascular dementia for example.

Acknowledgements. This work is supported by the EPSRC-funded UCL Centre for Doctoral Training in Medical Imaging (EP/L016478/1), the Department of Healths NIHR-funded Biomedical Research Centre at University College London Hospitals, the Wolfson Foundation (PR/ylr/18575), MRC (UK) at University College London Hospitals NHS Foundation Trust (540649). and Alzheimer's Society UK (AS-PG-15-025).

\section{REFERENCES}

[1] Mead, S., "Prion disease genetics," European Journal of Human Genetics (14), 273-281 (2006).

[2] Bradford, B. M., Piccardo, P., Ironside, J. W., and Mabbott, N. A., "Human prion diseases and the risk of their transmission during anatomical dissection," Clinical Anatomy 27(6), 821-832 (2014).

[3] Zerr, I. and Poser, S., "Clinical diagnosis and differential diagnosis of CJD and vCJD With special emphasis on laboratory tests," Acta Pathologica, Microbiologica et Immunologica Scandinavica 110, 88-98 (2002).

[4] Schroter, A., Zerr, I., Henkel, K., Tschampa, H. J., Finkenstaedt, M., and Poser, S., "Magnetic Resonance Imaging in the Clinical Diagnosis of Creutzfeldt-Jakob Disease," Journal of American Medical Association 57, 1751-1757 (2000).

[5] Wadsworth, J. D. F., Hill, A. F., Beck, J. A., and Collinge, J., "Molecular and clinical classification of human prion disease," British Medical Bulletin (66), 241-254 (2003).

[6] Caobelli, F., Cobelli, M., Pizzocaro, C., Pavia, M., Magnaldi, S., and Guerra, U. P., "The Role of Neuroimaging in Evaluating Patients Affected by Creutzfeldt-Jakob Disease: A Systematic Review of the Literature.," Journal of neuroimaging : official journal of the American Society of Neuroimaging 6, 1-12 (2014).

[7] Manix, M., Kalakoti, P., Henry, M., Thakur, J., Menger, R., Guthikonda, B., and Nanda, A., "CreutzfeldtJakob disease: updated diagnostic criteria, treatment algorithm, and the utility of brain biopsy," Neurosurgical Focus 39(November), 1-11 (2015).

[8] Johnson, R. T., "Prion diseases," Lancet Neurology 4, 635-42 (2005).

[9] Cardoso, M. J., Modat, M., Wolz, R., Melbourne, A., Cash, D., Rueckert, D., and Ourselin, S., "Geodesic Information Flows: Spatially-Variant Graphs and Their Application to Segmentation and Fusion," IEEE Transactions on Medical Imaging 34(9), 1976-1988 (2015).

[10] Shenton, M. E., Hamoda, H. M., Schneiderman, J. S., Bouix, S., Pasternak, O., Rathi, Y., Vu, M.-a., Purohit, M. P., Helmer, K., Koerte, I., Lin, a. P., Westin, C.-F., Kikinis, R., Kubicki, M., Stern, R. a., and Zafonte, R., "A review of magnetic resonance imaging and diffusion tensor imaging findings in mild traumatic brain injury.," Brain imaging and behavior 6, 137-92 (jun 2012).

[11] Sudre, C., Cardoso, M. J., Bouvy, W., Biessels, G., Barnes, J., and Ourselin, S., "Bayesian model selection for pathological neuroimaging data applied to white matter lesion segmentation," Medical Imaging, IEEE Trans. On (TMI) 34(c), 1-1 (2015). 